\title{
Efficacy of WBV as a modality for inducing changes in body composition, aerobic fitness, and muscular strength: a pilot study
}

This article was published in the following Dove Press journal:

Clinical Interventions in Aging

23 December 2013

Number of times this article has been viewed

\author{
Lauren R Tapp ${ }^{1,3}$ \\ Joseph F Signorile 1,2,4 \\ 'Departments of Kinesiology and \\ Sport Sciences, Coral Gables, ${ }^{2}$ Center \\ on Aging, University of Miami Miller \\ School of Medicine, Miami, FL, \\ ${ }^{3}$ Department of Kinesiology, Temple \\ University, Philadelphia, PA, ${ }^{4}$ Geriatric \\ Research, Education, and Clinical \\ Center, Bruce W Carter Department \\ of Veterans Affairs Medical Center, \\ Miami, FL, USA
}

\begin{abstract}
The purpose of this pilot study was to evaluate the effectiveness of whole body vibration (WBV) training as a modality for inducing changes in body composition, cardiovascular condition, and muscular strength in sedentary postmenopausal women. WBV training was compared with other training regimens, ie, aerobic training and circuit resistance training, commonly used to promote weight loss, cardiovascular conditioning, and muscular strength. Postmenopausal women (aged 48-60 years) were randomly assigned to WBV training, circuit resistance training, or aerobic training. Participants trained three times per week for 8 weeks. The training regimens were progressive in nature, with increases in training intensity and duration occurring throughout the 8-week period. Body composition was assessed using dual-energy $\mathrm{X}$-ray absorptiometry analyses. A modified Bruce treadmill protocol was used to assess aerobic capacity $\left(\mathrm{VO}_{2 \text { peak }}\right)$ and time to peak exhaustion. Upper and lower body strengths were determined by one repetition maximum (1-RM) chest and leg presses, respectively. Variables were analyzed using separate 3 (exercise mode) $\times 2$ (time) repeated-measures analysis of variance with effect sizes due to the small sample size. No significant main effects or interactions were seen for any body composition variable; however, moderate to large effect sizes $\left(\eta^{2}=0.243\right.$ and $\left.\eta^{2}=0.257\right)$ were detected regarding interactions for percent body fat and lean body mass favoring aerobic training and circuit resistance training. For $\mathrm{VO}_{2 \text { peak }}$, no significant main effects or interactions were detected (time, $\eta^{2}=0.150 ; P=0.11$; time $\times$ group, $\eta^{2}=0.139 ; P=0.30$ ); but a significant time effect was observed for time to peak exhaustion $\left(\eta^{2}=0.307 ; P=0.017\right)$. A significant interaction for upper body strength $\left(\eta^{2}=0.464 ; P=0.007\right)$, and main effect for time in lower body strength $\left(\eta^{2}=0.663 ; P=0.0001\right)$ was detected. Post hoc analysis indicated a significant increase in upper body strength for circuit resistance training $(P=0.023)$ and a decrease for WBV training $(P=0.015)$. Our results indicate that WBV may not be an effective alternative to traditional training with regard to body composition or aerobic capacity, but could have a positive impact on lower body strength.
\end{abstract}

Keywords: acceleration training, percent body fat, lean body mass, exercise, maximum oxygen consumption

\section{Introduction}

Obesity contributes significantly to a number of health conditions, including cardiovascular and metabolic diseases, and maintaining a healthy weight is associated with optimal health. A healthy body weight may be achieved through a combination of exercise and a healthy diet. ${ }^{1-3}$ Despite these established concepts, and the increasing availability of weight loss products and programs, the incidence of obesity continues to escalate.
Correspondence: Joseph F Signorile University of Miami, PO Box 248065,

Coral Gables, FL 33124, USA

$\mathrm{Tel}+\mathrm{I} 305898$ I457

$\mathrm{Fax}+\mathrm{I} 3052845168$

Email jsignorile@miami.edu 
One problem that exists for many overweight and obese individuals is an inability to perform exercise for a duration and intensity sufficient to induce changes in body weight or body composition. ${ }^{4,5}$ Weight-related issues, such as orthopedic limitations or reduced physical capacity, make it difficult for overweight or obese individuals to perform many of the traditional land-based exercise activities. These limitations, in conjunction with time constraints, lack of enjoyment in performing regular exercise, and work or family issues, create reasons to avoid regular exercise programs. Because of these factors, many individuals begin exercise programs but have difficulty with adherence, and eventually stop exercising. ${ }^{6}$ Evidence exists suggesting that adherence rates are lower when exercise sessions are longer, particularly when comparing 15-minute or 30-minute sessions with 45-minute sessions. ${ }^{7}$ Since whole body vibration exercise sessions are typically shorter in duration, this modality may promote greater adherence.

Postmenopausal women are at higher risk for increases in body fat and decreases in muscle mass and bone density than younger women and men. ${ }^{8-11}$ The increased risks are likely due to contributions from several factors, including decreased physical activity and lower estrogen levels. ${ }^{12}$ Increased body fat also places postmenopausal women at increased risk for development of cardiovascular disease, diabetes, and cancer. ${ }^{13}$

In order to overcome the actual or perceived barriers associated with traditional exercise, new modalities with the potential to impact body weight and composition must be investigated. One such modality is whole body vibration (WBV) exercise. Existing research indicates that there may be potential for WBV to positively impact body composition and muscular strength. ${ }^{1,14-19}$ For example, three separate studies have reported a relationship between vibration exercise and oxygen consumption and/or energy expenditure, ${ }^{20-22}$ with one report finding that WBV training increases oxygen consumption at a rate similar to that of moderate walking. ${ }^{20}$ Therefore, WBV may serve as an alternative modality for individuals who cannot or prefer not to participate in traditional modes of exercise.

In addition to the potential for improving body composition, WBV has been shown to induce positive changes in neuromuscular performance, such as increased strength and power output. ${ }^{18,22-24}$

To date, only one study by Roelants et al has examined the effects of WBV on body composition..$^{22}$ Although these researchers found that 24 weeks of WBV training did not produce any significant changes in body composition in young, untrained females, the exercise training protocol lacked a key element necessary to increase oxygen consumption, ie, external load, as indicated by three published studies, and a pilot study from our laboratory. ${ }^{20,21,25,26}$ Further, a protocol designed to assess the effects of WBV exercise on an older, more sedentary population would have considerable value given the potential application in managing sarcopenic obesity.

The purpose of this study was to compare the impact of a progressive WBV exercise program with that of two traditional programs (circuit resistance training and aerobic training) that have been shown to have positive effects on body composition in postmenopausal women. ${ }^{1,14-17} \mathrm{We}$ hypothesized that WBV exercise would have an effect on body composition similar to that of the traditional methods of aerobic and circuit training. However, due to the shorter workout times and unique environment associated with WBV exercise compared with aerobic training and circuit training, WBV exercise may provide a viable alternative to these more traditional forms of exercise, thereby effectively promoting physical activity through a greater adherence rate.

\section{Materials and methods Subjects}

Subjects were recruited using flyers distributed throughout the university's medical campus, and by advertisements placed in campus and community print and electronic media. The initial contact with subjects occurred by either telephone or electronic mail to introduce the study parameters and assess participant eligibility. This was followed by an inperson interview with subjects who remained interested and met the inclusion criteria. Prior to participation, all subjects completed an eligibility questionnaire, a medical screening form, and a consent form approved by the university's subcommittee for the use and protection of human subjects.

In order to be considered for the study, potential subjects had to be healthy, sedentary females aged $48-65$ years and with a body mass index $(\mathrm{BMI}) \geq 24$. Major exclusion criteria were current smoking, current use of hormone replacement therapy, and current or recent (within the past year) participation in WBV training, or any other exercise training program. Participants who met the initial eligibility requirements as determined by the telephone questionnaire were given details of the study, including exactly what their participation would entail, as well as frequency and duration of each appointment. Participants were told that although they would not receive any monetary compensation, they would receive two dual-energy X-ray absorptiometry (DEXA) scans, two complete fitness 
evaluations, and 8 weeks of exercise training with a fitness professional. Additionally, they were informed that their participation in the study would be purely voluntary and that they were free to discontinue their involvement at any time.

Of the 28 enrolled participants, 19 completed all program requirements. Six of the enrolled participants withdrew for various reasons, including work conflicts $(n=3)$, aggravation of previous injuries $(n=2)$, and transport problems $(n=1)$. Three participants did not attend for their first training session, despite completing all baseline testing.

\section{Study design}

This prospective, training study examined the effectiveness of WBV exercise as an effective intervention for weight loss, aerobic capacity, and strength compared with the established training strategies of aerobic training and circuit resistance training. All exercise protocols were progressive in nature and were designed to reflect training strategies normally practiced by individuals desiring to induce these changes. Group and time effects and time $\times$ group interactions for the response variables were assessed using repeated-measures analysis of variance and, due to the small sample size, effect sizes were also reported. During the training period, subjects were asked to refrain from additional physical activity and were told to follow their normal eating habits.

\section{Testing procedures}

Once participants completed the initial screening process and met the eligibility criteria, they reported at the laboratory for baseline testing. A verbal and written explanation of all test procedures was provided, and each participant was again given the opportunity to ask questions. The evaluations included standard height and weight measurements (Healthometer 402 EXP Doctor's Scale, Bridgeview, IL, USA), resting heart rate and blood pressure, and completion of the health screening questionnaire. Following this initial screening, baseline testing was continued.

\section{Body composition}

Participants were scheduled to receive a DEXA scan within 1 week of the initial testing day. A trained and licensed technician performed all DEXA scans. DEXA reports included a detailed assessment of body composition, including body weight, BMI, percent body fat, and lean body mass.

\section{Aerobic capacity}

On a third testing day, participants were given a baseline aerobic capacity treadmill test. Due to the practical considerations of performing a maximal aerobic capacity test, a peak oxygen consumption $\left(\mathrm{VO}_{2 \text { peak }}\right)$ test was chosen. All metabolic equipment was calibrated prior to each treadmill test and participants were fitted with a gas collection mask and heart rate monitor (model F51, Polar USA, Lake Success, NY, USA), which transmitted a constant heart rate response to the metabolic cart computer. Gas exchange data were collected and analyzed using the Viasys metabolic cart (Oxycon Mobile, Viasys, Yorba Linda, CA, USA). The participant was provided with a 3-minute warm-up period which also acquainted her with the treadmill (Quinton Treadmills, Bothell, WA, USA). During the warm-up period, the test procedures were again explained and the participants were informed that they would periodically be asked to provide their perceived level of exertion by selecting a number between 6 and 20 from a visual chart. ${ }^{27}$ The $\mathrm{VO}_{2 \text { peak }}$ test required the participant to walk on a treadmill at a pace consistent with the modified Bruce protocol. ${ }^{28}$ Participants were instructed to request to stop when they reached maximum volitional fatigue, indicated by reaching a rating of perceived exertion $\geq 17$ on the Borg scale of 6-20. An active cool-down period of 3-5 minutes was administered following the completion of the test. Time to peak exhaustion was also recorded.

\section{I-RM strength testing}

After an appropriate break of approximately 5-10 minutes following the aerobic capacity test, participants performed one-repetition maximum (1-RM) strength tests for the upper body and lower body, using chest press and leg press, respectively, on selectorized exercise machines (Life Fitness Circuit Series, Schiller Park, IL, USA). Participants were shown how to perform each exercise with proper form. Seat settings were adjusted appropriately for each participant to ensure optimal body and joint positioning. The leg press exercise position consisted of the participant being seated in a reclined position with feet placed on the platform slightly wider than hip width, and knees held at $90^{\circ}$. The chest press exercise required the participant to sit with her back against the pad and feet flat on the floor. Hands were positioned such that they were adjacent to the chest. Seat settings were recorded in order to maintain consistency for post-testing. Testing of the 1-RM consisted of completing three warm-up sets of ten, five, and three repetitions using a comfortable weight, increasing the weight for each set. ${ }^{29,30}$ Once the warm-up sets were completed, weight was progressively increased for repeated single repetition attempts until failure occurred. A rest period of 3-5 minutes was allowed between 
maximum attempts. The 1-RM score was recorded as the highest weight successfully lifted.

\section{Post-testing}

Within 1 week of completion of the 8-week exercise training program, all participants returned to the research laboratory for post-testing. Post-testing appointment times were scheduled as close to the pretesting appointment time as possible.

\section{Training protocols}

\section{Randomization}

Following baseline testing, participants were randomly assigned to one of three training modalities, ie, WBV training, aerobic training, or circuit resistance training. Participants remained in these respective groups for the duration of the study, thus each participant completed only one type of exercise training program. Regardless of group assignment, participants were required to complete three exercise sessions per week for 8 weeks, for a total number of 24 sessions. Because all three protocols were progressive in nature, including an increase in duration of exercise sessions, the total time per session and per week varied from week to week. During the final week, ie, week 8, WBV sessions were approximately 17-20 minutes in duration, while aerobic training and circuit resistance training sessions lasted approximately 45 minutes. Thus, on a weekly basis, WBV training was performed for roughly 60 minutes, versus 2.25 hours for aerobic training and circuit resistance training.

\section{WBV training protocol}

WBV training involved multiple sets of a squat exercise on a Power Plate vibration platform (Power Plate Pro5, Power Plate North America, Northbrook, IL, USA). Squats were performed in sets of ten, 15 , or 20 repetitions (or 30, 45, or 60 seconds per set, respectively) at a rate of one squat every 3 seconds. Participants were carefully monitored to ensure proper form. They were instructed to stand on the vibration platform with feet slightly wider than shoulder width apart. Each participant was directed to squat as deeply as she could up to a knee angle of $1.57 \mathrm{rad}$, making sure to maintain heel contact with the platform throughout the movement. Participants rested their hands on the console for balance, but were instructed to maintain an open handgrip to reduce their capacity to use the console for help during the exercise. During the latter stages of the protocol, external loads were applied using a weighted belt.

A periodized strategy allowed variation in training sets and repetitions during WBV training, thereby adding variety and reducing boredom. The training protocol was also progressively altered by applying external load, as a percent of lean body mass, and increasing exercise duration (see Table 1). A work to recovery ratio of 1:1 was maintained throughout the 8-week program. Participants were given an introductory period which consisted of low volume and low vibration frequency. The original protocol design included 1 week of low-amplitude vibration, with the intention of increasing the vibration amplitude. As participants became acquainted with WBV training, the amplitude was intended to increase significantly; however, some participants reported unacceptable discomfort with highamplitude vibration. Therefore, the protocol was redesigned to include only low-amplitude vibration.

The periodized model was based on weekly variation, and applied undulating durations of work periods while maintaining total volume. This strategy has not yet been documented as the optimal approach for performing WBV exercise; however, it allowed cycles of relative overload and recovery and added a degree of variety that was, anecdotally, less monotonous for the exercising participants.

\section{Aerobic training protocol}

Participants who were assigned to the aerobic training group performed an 8-week progressive treadmill-walking program in accordance with American College of Sports

Table I Whole body vibration training protocol

\begin{tabular}{|c|c|c|c|c|}
\hline Session & $\begin{array}{l}\text { Frequency } \\
\text { (Hz) }\end{array}$ & $\begin{array}{l}\text { Load } \\
\text { (\% LBM) }\end{array}$ & $\begin{array}{l}\text { Sets/ } \\
\text { repetitions }\end{array}$ & $\begin{array}{l}\text { Time/set } \\
\text { (seconds) }\end{array}$ \\
\hline $1-3 *$ & 30 & 0 & $4 / 10$ & 30 \\
\hline 4 & 35 & 5 & $6 / 10$ & 30 \\
\hline 5 & 35 & 5 & $4 / 15$ & 45 \\
\hline 6 & 35 & 5 & $3 / 20$ & 60 \\
\hline 7 & 35 & 10 & $7 / 10$ & 30 \\
\hline 8 & 35 & 10 & $5 / 15$ & 45 \\
\hline 9 & 35 & 10 & $4 / 20$ & 60 \\
\hline 10 & 35 & 15 & $8 / 10$ & 30 \\
\hline II & 35 & 15 & $6 / 15$ & 45 \\
\hline 12 & 35 & 15 & $5 / 20$ & 60 \\
\hline 13 & 40 & 20 & $9 / 10$ & 30 \\
\hline 14 & 40 & 20 & $7 / 15$ & 45 \\
\hline 15 & 40 & 20 & $6 / 20$ & 60 \\
\hline 16 & 40 & 20 & $10 / 10$ & 30 \\
\hline 17 & 40 & 25 & $8 / 15$ & 45 \\
\hline 18 & 40 & 25 & $7 / 20$ & 60 \\
\hline 19 & 40 & 25 & $11 / 10$ & 30 \\
\hline 20 & 40 & 25 & $9 / 15$ & 45 \\
\hline 21 & 40 & 30 & $8 / 20$ & 60 \\
\hline 22 & 40 & 30 & $12 / 10$ & 30 \\
\hline 23 & 40 & 30 & $10 / 15$ & 45 \\
\hline 24 & 40 & 30 & $9 / 20$ & 60 \\
\hline
\end{tabular}

Note: *Sessions I-3 were familiarization sessions. Abbreviation: \% LBM, percent load of lean body mass. 
Medicine guidelines. ${ }^{31}$ Participants walked on a treadmill (Life Fitness) on 3 nonconsecutive days per week for 8 weeks. The intensity and duration of the aerobic training program increased progressively throughout the 8-week study. Intensity was defined as percent of age-predicted maximal heart rate.

All exercise sessions began with a 5-minute warm-up at approximately $40 \%$ of age-predicted maximal heart rate and ended with a 5-minute cool-down at a continually declining intensity. Exercise intensity was monitored throughout the exercise sessions using a Polar heart rate monitor.

A trained and certified exercise professional was present at all training sessions to ensure safety and protocol adherence. In order to achieve and maintain appropriate exercise intensity, treadmill speed and grade were adjusted to keep the participant at her appropriate training heart rate. The aerobic training protocol is outlined in Table 2 .

\section{Circuit resistance training protocol}

The first circuit resistance training session consisted of participants completing additional 1-RM testing on all nine selectorized exercise machines (Life Fitness) to determine the exercise intensity that would be used for the 8-week training program. The nine exercises that were performed included, in the following order: leg press, chest press, leg extension, lat pulldown, seated leg curl, shoulder press, biceps curl, triceps extension, and abdominal crunch. Exercise sessions were performed on 3 nonconsecutive days per week. The intensity of the exercise was initially set at $50 \%$ of the 1-RM, and increased to $55 \% 1-\mathrm{RM}$ and $60 \% 1-\mathrm{RM}$ at weeks 4 and 6, respectively, as part of the progressive strategy. Total volume of exercise gradually increased over the 8-week period as rest/transition periods were decreased (Table 3). A trained and certified fitness professional was present at all training sessions for safety and to ensure protocol adherence.

Table 2 Aerobic training protocol

\begin{tabular}{lll}
\hline Session \# & Intensity & Duration (minutes) \\
\hline $1-3 *$ & $50 \%-60 \%$ & 20 \\
4 & $60 \%-65 \%$ & 20 \\
$5-6$ & $60 \%-65 \%$ & 25 \\
$7-9$ & $65 \%-70 \%$ & 30 \\
$10-12$ & $65 \%-70 \%$ & 35 \\
$13-14$ & $70 \%-75 \%$ & 30 \\
$15-16$ & $70 \%-75 \%$ & 35 \\
$17-18$ & $70 \%-75 \%$ & 40 \\
$19-2 \mid$ & $75 \%-80 \%$ & 35 \\
$22-24$ & $75 \%-80 \%$ & 40 \\
\hline
\end{tabular}

Notes: Intensity was determined by age-predicted maximal heart rate. *Sessions I-3 were familiarization sessions.
Table 3 Circuit resistance training protocol

\begin{tabular}{llll}
\hline Session & Sets/repetitions & Intensity & Rest period (seconds) \\
\hline I* & - & $100 \%$ & - \\
$2-3$ & $1 / 12$ & $50 \%$ & 60 \\
$4-9$ & $2 / 12$ & $50 \%$ & 30 \\
$10-12$ & $2 / 12$ & $55 \%$ & 45 \\
$13-15$ & $3 / 12$ & $55 \%$ & 60 \\
$15-18$ & $3 / 12$ & $55 \%$ & 30 \\
$19-24$ & $3 / 12$ & $60 \%$ & 45 \\
\hline
\end{tabular}

Notes: Intensity was determined by a I-RM test procedure. *Session I was used to obtain I-RM.

Abbreviation: I-RM, one repetition maximum.

\section{Statistical analyses}

Descriptive data (ie, age, height, weight, and BMI) were analyzed using one-way analysis of variance. Separate $3 \times 2$ repeated-measures analysis of variance, with three levels of exercise mode (WBV training, aerobic training, and circuit resistance training), and two levels of time (pretest and posttest) were initially used to analyze the main outcome variables: body weight, BMI, percent body fat, lean body mass, upper and lower body 1-RM (using the chest press and leg press, respectively), $\mathrm{VO}_{2 \text { peak }}$, and time to peak exhaustion. Due to the small sample size, all analyses included quantifications of effect size. Analyses were performed using PASW software version 18 (SPSS Inc., Chicago, IL, USA).

\section{Results}

\section{Participant characteristics}

The physical characteristics of the study participants $(n=19)$ are presented in Table 4.

\section{Body composition}

No significant pretraining differences were observed between the groups for age, height, body weight, BMI, percent body fat, or lean body mass. Mean values, change scores, and percent difference for body weight, BMI, percent body fat, and lean body mass are presented in Table 5 . The $3 \times 2$ repeatedmeasures analysis of variance revealed no significant main

Table 4 Physical characteristics of subjects

\begin{tabular}{lccc}
\hline & $\begin{array}{l}\text { WBVT } \\
(\mathbf{n}=6)\end{array}$ & $\begin{array}{l}\text { AT } \\
(\mathbf{n}=6)\end{array}$ & $\begin{array}{l}\text { CT } \\
(\mathbf{n}=7)\end{array}$ \\
\hline Age (years) & $53.2 \pm 2.1$ & $55.2 \pm 6.4$ & $54.1 \pm 5.3$ \\
Height $(\mathrm{m})$ & $1.64 \pm 0.06$ & $1.60 \pm 0.07$ & $1.61 \pm 0.07$ \\
Weight $(\mathrm{kg})$ & $87.73 \pm 23.69$ & $90.00 \pm 8.27$ & $79.00 \pm 8.27$ \\
BMI & $32.6 \pm 7.9$ & $35.3 \pm 5.6$ & $30.6 \pm 3.2$ \\
\hline
\end{tabular}

Note: Values are the mean \pm standard deviation.

Abbreviations: BMI, body mass index; CT, circuit resistance training; AT, aerobic training; WBVT, whole body vibration training. 
Table 5 Body composition

\begin{tabular}{lllll}
\hline & Pre & Post & $\Delta$ & $\begin{array}{l}\text { Percent } \\
\text { change }\end{array}$ \\
\hline W $_{\text {B }}(\mathrm{kg})$ & & & & \\
WBVT & $87.73 \pm 23.69$ & $88.07 \pm 23.30$ & 0.34 & 0.39 \\
AT & $90.00 \pm 8.27$ & $90.08 \pm 8.27$ & 0.08 & 0.09 \\
CT & $79.00 \pm 8.27$ & $80.00 \pm 7.37$ & 1.01 & 1.28 \\
BMI & & & & \\
WBVT & $32.55 \pm 7.93$ & $32.65 \pm 7.68$ & 0.10 & 0.31 \\
AT & $35.28 \pm 5.60$ & $35.35 \pm 5.69$ & 0.07 & 0.19 \\
CT & $30.61 \pm 3.24$ & $31.06 \pm 3.23$ & 0.45 & 1.47 \\
BF\% & & & & \\
WBVT & $46.47 \pm 5.16$ & $47.12 \pm 4.57$ & 0.65 & 0.13 \\
AT & $52.63 \pm 5.62$ & $52.13 \pm 5.88$ & -0.50 & -0.01 \\
CT & $44.61 \pm 5.49$ & $44.37 \pm 5.66$ & -0.24 & -0.01 \\
LBM $(k g)$ & & & & \\
WBVT & $46.18 \pm 9.21$ & $45.81 \pm 8.67$ & -0.36 & 0.78 \\
AT & $42.46 \pm 3.60$ & $42.85 \pm 3.8 \mathrm{I}$ & 0.49 & 1.15 \\
CT & $43.59 \pm 5.000$ & $44.37 \pm 4.92$ & 0.78 & 1.78 \\
\hline
\end{tabular}

Note: Values are the mean \pm standard deviation.

Abbreviations: $\mathrm{BMI}$, body mass index; $\mathrm{W}_{\mathrm{B}}$, body weight; $\mathrm{BF} \%$, percent body fat; LBM, lean body mass; CT, circuit resistance training; AT, aerobic training; WBVT, whole body vibration training.

effects or interactions for any of the four variables. However, for percent body fat, a moderate effect size was seen for the time $\times$ group interaction $\left(\eta^{2}=0.243 ; P=0.10\right)$, with the WBV group showing a slight increase in percent body fat while the aerobic training and circuit resistance training groups showed minor declines. A time $\times$ group interaction also approached significance for lean body mass $(P=0.093)$. As can be seen from the results reported in Table 5, there was a slight decline in lean body mass for the WBV group, while the aerobic training and circuit resistance training groups showed minor increases. The effect size for the time $\times$ group interaction for lean body mass was moderate to large $\left(\eta^{2}=0.257\right)$.

\section{Aerobic capacity}

The results for $\mathrm{VO}_{2 \text { peak }}$ and time to peak exhaustion are presented in Table 6 . The $3 \times 2$ repeated-measures analysis of variance for $\mathrm{VO}_{2 \text { peak }}$ revealed no significant main effect for time $\left(\eta^{2}=0.150 ; P=0.11\right)$ or time $\times$ group interaction $\left(\eta^{2}=0.139 ; P=0.30\right)$. For time to peak exhaustion, a significant main effect for time was observed $\left(\eta^{2}=0.307 ; P=0.017\right)$.

\section{I-RM strength}

Results for upper body and lower body strengths are presented in Table 7 . The $3 \times 2$ repeated-measures analysis of variance revealed a significant interaction for upper body strength $\left(\eta^{2}=0.464 ; P=0.007\right)$, and for lower body strength, a main effect for time $\left(\eta^{2}=0.663 ; P=0.0001\right)$. Post hoc analysis
Table 6 Aerobic capacity

\begin{tabular}{lrlll}
\hline \multicolumn{2}{c}{ Pre } & Post & $\Delta$ & $\begin{array}{l}\text { Percent } \\
\text { change }\end{array}$ \\
\hline $\mathrm{VO}_{\text {2peak }}(\mathrm{mL} / \mathrm{kg} / \mathrm{min})$ & & & & \\
WBVT & $19.15 \pm 3.88$ & $18.50 \pm 2.38$ & -0.65 & 3.39 \\
AT & $16.27 \pm 3.12$ & $19.38 \pm 3.39$ & 3.11 & 19.11 \\
CT & $16.41 \pm 6.10$ & $19.24 \pm 5.99$ & 2.83 & 17.25 \\
TPE (minutes) & & & & \\
WBVT & $12.32 \pm 2.62$ & $12.71 \pm 2.20$ & 0.39 & 3.17 \\
AT & $9.36 \pm 1.45$ & $11.39 \pm 1.81$ & 2.03 & 21.69 \\
CT & $11.94 \pm 2.49$ & $13.21 \pm 2.25$ & 1.27 & 10.64 \\
\hline
\end{tabular}

Notes: $*$ Significant effect by time $(P=0.017)$. WBVT $(n=6)$, AT $(n=6), C T(n=7)$. Abbreviations: TPE, time to peak exhaustion; $C T$, circuit resistance training; $\mathrm{AT}$, aerobic training; $\mathrm{WBVT}$, whole body vibration training; $\mathrm{VO}_{\text {2peak }}$, peak oxygen uptake.

indicated a significant increase in upper body strength in the circuit resistance training group $(P=0.023)$ and a significant decrease in the WBV group $(P=0.015)$. One-way analysis of variance performed for change in scores for upper body strength showed a significant difference between WBV training and circuit resistance training $(P=0.005)$.

\section{Discussion}

The primary objective of this study was to determine if 8 weeks of WBV training could produce positive changes in body composition, muscular strength, and aerobic capacity compared with those produced by aerobic training and circuit resistance training. Our results indicate that WBV training had no effect on body composition; however, there is some evidence that WBV training may have the potential to positively impact aerobic performance and muscular strength. As expected, circuit resistance training resulted in the greatest improvements in 1-RM scores for the upper body, while WBV training, circuit resistance training, and aerobic training

Table 7 I-RM strength

\begin{tabular}{|c|c|c|c|c|}
\hline & Pre & Post & $\Delta$ & $\begin{array}{l}\text { Percent } \\
\text { change }\end{array}$ \\
\hline \multicolumn{5}{|l|}{ UBRM (kg) } \\
\hline WBVT & $18.13 \pm 8.95$ & $16.04 \pm 7.10$ & -2.08 & $11.49 *$ \\
\hline AT & 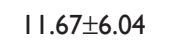 & $11.88 \pm 5.13$ & 0.21 & 1.78 \\
\hline CT & $16.96 \pm 7.17$ & $18.75 \pm 6.56$ & 1.79 & $10.54 *$ \\
\hline \multicolumn{5}{|c|}{ LBRM $(\mathrm{kg})^{* *}$} \\
\hline WBVT & $101.14 \pm 45.40$ & $120.83 \pm 38.20$ & 19.70 & 19.47 \\
\hline AT & $62.50 \pm 19.8$ & $75.38 \pm 31.54$ & 12.88 & 20.60 \\
\hline $\mathrm{CT}$ & $80.20 \pm 19.9$ & $94.15 \pm 16.33$ & 13.96 & 17.41 \\
\hline
\end{tabular}

Notes: WBVT $(n=6)$, AT $(n=6)$, CT $(n=7)$. *Significant within group difference $(P<0.05)$; **significant main effect for time.

Abbreviations: UBRM, upper body one repetition maximum using the chest press; LBRM, lower body one repetition maximum using the leg press; I-RM, one repetition maximum; $\mathrm{CT}$, circuit resistance training; AT, aerobic training; WBVT, whole body vibration training. 
induced similar positive changes in lower body strength. All three training modalities resulted in significant improvements in time to peak exhaustion.

\section{Body composition}

The results of the current study do not support the use of 8 weeks of WBV training as an effective intervention for producing positive changes in body composition in obese postmenopausal women. This observation is in agreement with the findings of Roelants et al who found no significant changes in percent body fat after 24 weeks of WBV training in untrained women aged 20.3 \pm 2.0 years. ${ }^{22}$ Our results also reflect those reported by von Stengel et al, who saw no additional benefit when adding WBV to 15 minutes of lower body strength training when performed using a protocol including 20 minutes of aerobic dance (70-80/HRmax), 5 minutes of balance and coordination training, and 20 minutes of upper and lower body elastic band training. ${ }^{32}$ Verschueren et al found no significant changes in lean body mass after 6 months of WBV training, which supports the lack of improvement in lean body mass in the current 8 -week study. ${ }^{33}$

Our results differ from those reported by Fjeldstad et al, who noted a significantly greater decrease in percent body fat over an 8-month training period for their WBV group compared with a resistance training and control group; however, the WBV and resistance training groups showed significant increases in bone-free lean body mass. ${ }^{34}$ Our results also vary to some extent from those reported by Lamont et al in men aged 20-30 years. ${ }^{35}$ These researchers reported a significantly greater increase in lean body mass than in controls for a group that performed WBV exercises during recovery from active squatting, rather than during the exercise itself. The exercise group that squatted without WBV recovery did not achieve significant increases in lean body mass above controls. In agreement with our results, only a group effect was seen for percent body fat; however, an alternative evaluation of change in scores revealed that only the squat group employing the WBV recovery showed a significant reduction in body fat. These data argue for utilization of WBV as a recovery method rather than a modality superimposed during a bout of standard resistance exercise. Also in contrast with our results, Vissers et al reported that 6 months of WBV training or aerobic training each had the capacity to reduce visceral adipose tissue and maintain that loss for the remainder of a year-long testing period, but only when combined with caloric restriction. ${ }^{36}$

There are a number of possible explanations for the lack of significant changes in body composition as a result of
WBV training seen in the current study. First of all, there is the matter of sample size. It should be noted that although there was no significant effect of WBV training on lean body mass in the current study, a moderate to large effect size was demonstrated $\left(\eta^{2}=0.257\right)$. Perhaps replicating the study with a larger number of subjects would yield significant values.

The second possibility is that the volume of work completed was not sufficient to produce a significant improvement in body composition. This argument is supported by the positive results seen by Fjeldstad et al in their 8-month study providing the WBV intervention three times per week in a sample of women aged $60-75$ years. ${ }^{34}$ Additionally, the reports by several investigators that acute WBV applied during dynamic exercise results in greater increases in oxygen uptake, ${ }^{22,37,38}$ and that WBV training can produce a significant increase in post-exercise energy expenditure, lend support to the potential for positive effects of this intervention on body composition when provided over a prolonged training period. ${ }^{37}$ However, the training results reported by Roelants et al, von Stengel et al, and Verschueren et al, as well as the excess post-exercise oxygen consumption results reported by Serravite et al, indicate that further research is warranted. ${ }^{22,26,32,33}$

A fourth issue when evaluating the effects of WBV training on body composition is the limited research to date on the topic, which makes it difficult to determine an optimal training protocol with which to adequately compare WBV training with aerobic training and circuit resistance training. We attempted to create a significant WBV training stimulus with progressive increases in exercise intensity throughout the duration of the study. The periodized protocol used in the current study varied the sets and repetitions, progressively increasing the volume of exercise on a weekly schedule. Because adding an external load to WBV training increases oxygen utilization rates, our protocol also used an external load $(10 \%-30 \%$ of lean body mass) to increase caloric expenditure. ${ }^{20,21,26}$ However, the conservative external load and WBV amplitude used may have been insufficient to positively impact percent body fat or lean body mass.

The conflicting results seen among these studies may best be summarized by the conclusions drawn by Cochrane in his review paper on the topic. Cochrane suggests that although WBV exercise does increase metabolic demand, even with the addition of external load and high frequency/ amplitude settings, it does not seem to match the intensity of traditional aerobic exercise necessary to induce changes in body composition. ${ }^{39}$ This is consistent with the findings of the current study, which shows that WBV training can 
provide some benefit, yet the ability to have an effect on body composition was not significant.

\section{Aerobic performance}

Aerobic performance was evaluated using $\mathrm{VO}_{2 \text { peak }}$ and time to peak exhaustion. Although aerobic training and circuit resistance training produced improvements in $\mathrm{VO}_{2 \text { peak }}$ of $19.1 \%$ and $17.3 \%$, respectively, these improvements were not statistically significant, and likely due to the small sample size. When comparing this result with the nonsignificant decline in $\mathrm{VO}_{2 \text { peak }}$ of $3.39 \%$ seen with WBV training, we question the relative effectiveness of WBV training in improving aerobic capacity compared with aerobic training and circuit resistance training.

For time to peak exhaustion, however, improvements were seen across all training modalities, with no significant differences between groups. As anticipated, the improvement in time to peak exhaustion scores using the aerobic training modality was the most pronounced (21.69\%), with circuit resistance training and WBV training producing progressively lower improvements $(10.64 \%$ and $3.15 \%$, respectively). Our results for aerobic training and circuit resistance training reflect the improvements in aerobic performance and reductions in fatigue previously reported in the literature with these training modalities. ${ }^{40-43}$

To date, there has been only a single investigation comparing the influence of WBV training and other training modalities on cardiovascular response. Bogaerts et al compared the effects of WBV training and a combined cardiovascular and resistance training program on cardiovascular fitness. ${ }^{44}$ They reported that both $\mathrm{VO}_{\text {2peak }}$ and time to peak exhaustion improved on WBV training and combined cardiovascular and resistance training groups of participants, with no significant differences between the groups. However, the standard program produced a greater improvement in time to peak exhaustion. Clearly, more controlled studies are required for effective examination of the potential for WBV training to improve not only oxygen consumption, but also other cardiovascular health factors.

\section{Maximum strength}

Upper body strength and lower body strength were assessed using the 1-RM test. As expected, the circuit resistance training exercise modality elicited a significant improvement in upper body strength for obese postmenopausal women, while WBV training had a significant negative effect. All three exercise modalities significantly increased lower body strength (between $17.4 \%$ and $20.6 \%$ ). The increase in upper body strength by circuit resistance training was attributable to inclusion of upper body exercises in the circuit resistance training protocol, whereas improvements in lower body strength were likely the result of all three training modalities requiring the use of lower body movements (ie, circuit resistance training participants performed specific lower body resistive training, aerobic training participants performed walking activity, and the WBV training participants performed squats as a part of their training). These data suggest that all three modalities of training may have beneficial effects on lower body strength. It has been well established in the literature that circuit resistance training improves lower body strength..$^{41,45-48}$

Our results are in agreement with a number of studies reporting that WBV training can be used to improve lower body strength in older persons. For example, two studies from the same research group showed that, in postmenopausal women, 24 weeks of WBV training produced similar improvements in static and dynamic strength as those produced by resistance training; however, the WBV training sessions lasted 10 minutes while the resistance training sessions lasted 40 minutes. ${ }^{23,33}$ A study by Bemben et al examined the impact of combining WBV training and high-intensity resistance training on 1-RM performance. ${ }^{49}$ They reported that the resistance training group improved over controls in 13 of 16 muscle groups tested, while WBV training plus resistance produced performance improvements in 15 of the 16 muscle groups. Bogaerts et al found comparative increases in leg extension strength and power between a mixed exercise program and WBV training for the upper and lower body muscles. ${ }^{44} \mathrm{~A}$ study by Rees et al found that a progressive WBV training protocol produced greater improvements in plantar flexion strength and similar knee and hip flexion and extension strength and power using a resistance training program without WBV. ${ }^{50}$ Machado et al found a significant increase in muscle strength and thigh muscle growth in women aged 65-90 years after ten weeks of selective squat exercises using vertical WBV training. ${ }^{51}$ Mikhael et al reported that vertical WBV training $(12 \mathrm{~Hz}, 1.0 \mathrm{~mm}$; 20 minutes per day, 3 days per week; 1-minute 1:1 work to recovery duty cycle) over a 3 -month training period improved upper body and lower body strength whether knees were in a locked or flexed condition..$^{52}$ Overall, these studies support our finding that a periodized WBV training program can positively affect strength and that these improvements may rival those produced by typical resistance training protocols. In addition, the improvements seen in lower body strength but not upper body strength in our study are likely the result of 
a decline in vibratory stimulus transmission across muscles and joints farther from the point of application. ${ }^{53,54}$

\section{Conclusion}

While the conclusions that can be drawn from this study are limited by the sample size, study duration, and potentially low resistance overload and volume used during WBV training, our analysis, including effect sizes, allows some conclusions to be drawn. The first is that none of the exercise protocols used in this study were effective in inducing positive changes in body composition measures across the 8 -week training period utilized. The second is that all of the training protocols appeared to be effective in improving test duration during treadmill testing, yet this was accomplished without significant improvements in $\mathrm{VO}_{2 \text { eak }}$. Finally, all the programs seemed effective in improving lower body strength, but improvements in upper body strength were limited to the resistance training group, which specifically targeted improvement in upper body strength using targeted exercises.

\section{Acknowledgments}

The authors would like to thank Dr Silvina Levis, Professor of Medicine and Director of Miller School of Medicine's Osteoporosis Center, and her staff for their help with the DEXA analyses, and Ms Gail Haldeman, Director of the Miller School of Medicine, and her staff for their collaboration, help with recruitment, and use of their training facility. We would also like to thank the following individuals from Temple University for their contributions and support throughout this project: Drs Zebulon Kendrick, Michael Sachs, Michael Brown, and Joseph DuCette.

\section{Disclosure}

The authors report no conflicts of interest in this work.

\section{References}

1. Shinkai S, Watanabe S, Kurokawa Y, Torii J, Asai H, Shephard RJ. Effects of 12 weeks of aerobic exercise plus dietary restriction on body composition, resting energy expenditure and aerobic fitness in mildly obese middle-aged women. Eur J Appl Physiol Occup Physiol. 1994;68(3):258-265.

2. Stefanick ML, Mackey S, Sheehan M, Ellsworth N, Haskell WL, Wood PD. Effects of diet and exercise in men and postmenopausal women with low levels of HDL cholesterol and high levels of LDL cholesterol. N Engl J Med. 1998;339(1):12-20.

3. Svendsen OL, Hassager C, Christiansen C. Impact of regional and total body composition and hormones on resting energy expenditure in overweight postmenopausal women. Metabolism. 1993;42(12):1588-1591.

4. King AC, Tribble DL. The role of exercise in weight regulation in nonathletes. Sports Med. 1991;11(5):331-349.

5. Wilson MA. Treatment of obesity. Am J Med Sci. 1990;299(1):62-68.
6. Perri MG, McAdoo WG, McAllister DA, et al. Enhancing the efficacy of behavior therapy for obesity. J Consult Clin Psychol. 1986;54(5): 670-675.

7. Pollock ML. Prescribing exercise for fitness and adherence. In: Dishman R, editor. Exercise Adherence: Its Impact On Public Health. Champaign, IL: Human Kinetics; 1988.

8. Bjorkelund C, Lissner L, Andersson S, Lapidus L, Bengtsson C. Reproductive history in relation to relative weight and fat distribution. Int J Obes Relat Metab Disord. 1996;20(3):213-219.

9. Poehlman ET, Toth MJ, Gardner AW. Changes in energy balance and body composition at menopause: a controlled longitudinal study. Ann Intern Med. 1995;123(9):673-675.

10. Toth MJ, Tchernof A, Sites CK, Poehlman ET. Effect of menopausal status on body composition and abdominal fat distribution. Int $J$ Obes Relat Metab Disord. 2000;24(2):226-231.

11. Tremollieres FA, Pouilles JM, Ribot CA. Relative influence of age and menopause on total and regional body composition changes in postmenopausal women. Am J Obstet Gynecol. 1996;175(6): 1594-1600.

12. Totosy de Zepetnek JO, Giangregorio LM, Craven BC. Whole-body vibration as potential intervention for people with low bone mineral density and osteoporosis: a review. J Rehabil Res Dev. 2009;46(4): 529-542.

13. Dennis KE. Postmenopausal women and the health consequences of obesity. J Obstet Gynecol Neonatal Nurs. 2007;36(5):511-517.

14. Asikainen TM, Miilunpalo S, Oja P, Rinne M, Pasanen M, Vuori I. Walking trials in postmenopausal women: effect of one vs two daily bouts of aerobic fitness. Scand J Med Sci Sports. 2002;12(2): 99-105.

15. Asikainen TM, Miilunpalo S, Oja P, et al. Randomised, controlled walking trials in postmenopausal women: the minimum dose to improve aerobic fitness? Br J Sports Med. 2002;36(3):189-194.

16. Brentano MA, Cadore EL, Da Silva EM, et al. Physiological adaptations to strength and circuit training in postmenopausal women with bone loss. J Strength Cond Res. 2008;22(6):1816-1825.

17. Brooke-Wavell K, Jones PR, Hardman AE. Brisk walking reduces calcaneal bone loss in post-menopausal women. Clin Sci (Lond). 1997;92(1):75-80.

18. Bosco C, Colli R, Introini E, et al. Adaptive responses of human skeletal muscle to vibration exposure. Clin Physiol. 1999;19(2):183-187.

19. Russo CR, Lauretani F, Bandinelli S, et al. High-frequency vibration training increases muscle power in postmenopausal women. Arch Phys Med Rehabil. 2003;84(12):1854-1857.

20. Rittweger J, Beller G, Felsenberg D. Acute physiological effects of exhaustive whole-body vibration exercise in man. Clin Physiol. 2000;20(2):134-142.

21. Rittweger J, Schiessl H, Felsenberg D. Oxygen uptake during wholebody vibration exercise: comparison with squatting as a slow voluntary movement. Eur J Appl Physiol. 2001;86(2):169-173.

22. Roelants M, Delecluse C, Goris M, Verschueren S. Effects of 24 weeks of whole body vibration training on body composition and muscle strength in untrained females. Int J Sports Med. 2004;25(1):1-5.

23. Roelants M, Delecluse C, Verschueren SM. Whole-body-vibration training increases knee extension strength and speed of movement in older women. J Am Geriatr Soc. 2004;52(6):901-908.

24. Torvinen S, Kannu P, Sievanen H, et al. Effect of a vibration exposure on muscular performance and body balance. Randomized cross-over study. Clin Physiol Funct Imaging. 2002;22(2):145-152.

25. Rittweger J, Ehrig J, Just K, Mutschelknauss M, Kirsch KA, Felsenberg D. Oxygen uptake in whole-body vibration exercise: influence of vibration frequency, amplitude, and external load. Int $J$ Sports Med. 2002;23(6):428-432.

26. Serravite DH, Roos BA, Mow S, Signorile JF. Increased oxygen consumption during whole body vibration in older men. Med Sci Sports Exerc. 2009;41(5):83.

27. Borg G. Perceived exertion as an indicator of somatic stress. Scand J Rehabil Med. 1970;2(2):92-98. 
28. Bruce RA, Blackmon JR, Jones JW, Strait G. Exercising testing in adult normal subjects and cardiac patients. Pediatrics. 1963;32 Suppl: $742-756$.

29. Hoeger WK, Hopkins DR, Barette SL, Hale DF. Relationship between repetitions and selected percentages of one repetition maximum: a comparison of untrained and trained males and females. J Appl Sports Sci Res. 1990;4(2):47-54.

30. Stone MH, O'Bryant HS. Weight Training: A Scientific Approach. Minneapolis, MN: Burgess; 1987.

31. American College of Sports Medicine. The recommended quantity and quality of exercise for developing and maintaining cardiorespiratory and muscular fitness in healthy adults. Med Sci Sports Exerc. 1998;30(6):975-991.

32. von Stengel S, Kemmler W, Engelke K, Kalender WA. Effect of wholebody vibration on neuromuscular performance and body composition for females 65 years and older: a randomized-controlled trial. Scand J Med Sci Sports. 2012;22(1):119-127.

33. Verschueren SM, Roelants M, Delecluse C, Swinnen S, Vanderschueren D, Boonen S. Effect of 6-month whole body vibration training on hip density, muscle strength, and postural control in postmenopausal women: a randomized controlled pilot study. $J$ Bone Miner Res. 2004;19(3):352-359.

34. Fjeldstad C, Palmer IJ, Bemben MG, Bemben DA. Whole-body vibration augments resistance training effects on body composition in postmenopausal women. Maturitas. 2009;63(1):79-83.

35. Lamont HS, Cramer JT, Bemben DA, Shehab RL, Anderson MA, Bemben MG. Effects of a 6-week periodized squat training with or without whole-body vibration upon short-term adaptations in squat strength and body composition. J Strength Cond Res. 2011;25(7):1839-1848.

36. Vissers D, Verrijken A, Mertens I, et al. Effect of long-term whole body vibration training on visceral adipose tissue: a preliminary report. Obes Facts. 2010;3(2):93-100.

37. Da Silva ME, Fernandez JM, Castillo E, et al. Influence of vibration training on energy expenditure in active men. $J$ Strength Cond Res. 2007;21(2):470-475.

38. Garatachea N, Jimenez A, Bresciani G, Marino NA, GonzalezGallego J, de Paz JA. The effects of movement velocity during squatting on energy expenditure and substrate utilization in whole-body vibration. J Strength Cond Res. 2007;21(2):594-598.

39. Cochrane DJ. Is vibration exercise a useful addition to a weight management program? Scand J Med Sci Sports. 2012;22(6):705-713.

40. Campos GE, Luecke TJ, Wendeln HK, et al. Muscular adaptations in response to three different resistance-training regimens: specificity of repetition maximum training zones. Eur J Appl Physiol. 2002; 88(1-2):50-60.

41. Gettman LR, Ayres JJ, Pollock ML, Jackson A. The effect of circuit weight training on strength, cardiorespiratory function, and body composition of adult men. Med Sci Sports. 1978;10(3):171-176.
42. Haltom RW, Kraemer RR, Sloan RA, Hebert EP, Frank K, Tryniecki JL. Circuit weight training and its effects on excess postexercise oxygen consumption. Med Sci Sports Exerc. 1999;31(11):1613-1618.

43. Tanaka H, Swensen T. Impact of resistance training on endurance performance. A new form of cross-training? Sports Med. 1998;25(3): 191-200.

44. Bogaerts AC, Delecluse C, Claessens AL, Troosters T, Boonen S, Verschueren SM. Effects of whole body vibration training on cardiorespiratory fitness and muscle strength in older individuals (a 1-year randomised controlled trial). Age Ageing. 2009;38(4):448-454.

45. Gettman LR, Pollock ML. Circuit training: a critical review of its physiological benefits. Phys Sports Med. 1981;9(1):44-60.

46. Gettman LR, Ward P, Hagan RD. A comparison of combined running and weight training with circuit weight training. Med Sci Sports Exerc. 1982;14(3):229-234.

47. Mosher PE, Underwood SA, Ferguson MA, Arnold RO. Effects of 12 weeks of aerobic circuit training on aerobic capacity, muscular strength, and body composition in college-age women. J Strength Cond Res. 1994;8(3):144-148.

48. Wilmore JH. Alterations in strength, body composition and anthropometric measurements consequent to a 10-week weight training program. Med Sci Sports. 1974;6(2):133-138.

49. Bemben DA, Palmer IJ, Bemben MG, Knehans AW. Effects of combined whole-body vibration and resistance training on muscular strength and bone metabolism in postmenopausal women. Bone. 2010;47(3):650-656.

50. Rees SS, Murphy AJ, Watsford ML. Effects of whole-body vibration exercise on lower-extremity muscle strength and power in an older population: a randomized clinical trial. Phys Ther. 2008; 88(4):462-470

51. Machado A, Garcia-Lopez D, Gonzalez-Gallego J, Garatachea N. Whole-body vibration training increases muscle strength and mass in older women: a randomized-controlled trial. Scand J Med Sci Sports. 2010;20(2):200-207.

52. Mikhael M, Orr R, Amsen F, Greene D, Singh MA. Effect of standing posture during whole body vibration training on muscle morphology and function in older adults: a randomised controlled trial. BMC Geriatr. 2010;10:74

53. Abercromby AF, Amonette WE, Layne CS, McFarlin BK, Hinman MR, Paloski WH. Vibration exposure and biodynamic responses during whole-body vibration training. Med Sci Sports Exerc. 2007; 39(10):1794-1800.

54. Pollock RD, Woledge RC, Mills KR, Martin FC, Newham DJ. Muscle activity and acceleration during whole body vibration: effect of frequency and amplitude. Clin Biomech (Bristol, Avon). 2010; 25(8):840-846
Clinical Interventions in Aging

\section{Publish your work in this journal}

Clinical Interventions in Aging is an international, peer-reviewed journal focusing on evidence-based reports on the value or lack thereof of treatments intended to prevent or delay the onset of maladaptive correlates of aging in human beings. This journal is indexed on PubMed Central, MedLine, the American Chemical Society's 'Chemical Abstracts Ser-
Dovepress

vice' (CAS), Scopus and the Elsevier Bibliographic databases. The manuscript management system is completely online and includes a very quick and fair peer-review system, which is all easy to use. Visit $\mathrm{http} / / / \mathrm{www}$. dovepress.com/testimonials.php to read real quotes from published authors. 